\title{
The Physical Nature of Length Contraction
}

\author{
—The DSSU* Theory of Length Contraction Induced by Absolute Motion
}

\author{
Conrad Ranzan ${ }^{1}$
}

${ }^{1}$ DSSU Research, Niagara Falls, Canada

Correspondence: Conrad Ranzan, Director, DSSU Research, 5145 Second Avenue, Niagara Falls, ON., L2E 4J8, Canada. Tel: 1-905-357-0788. E-mail: Ranzan@CellularUniverse.org; DSSUresearch4@aol.com

Received: October 8, 2012 Accepted: October 26, 2012 Online Published: January 9, 2013

doi:10.5539/apr.v5n1p87 URL: http://dx.doi.org/10.5539/apr.v5n1p87

\begin{abstract}
It is described how the mode by which matter is "conducted" through luminiferous aether causes the matter to contract. A simple derivation of the mathematical expression for this physical length contraction is presented. There is also a brief discussion of relevant historical aspects and of nonphysical length contraction.
\end{abstract}

Keywords: length contraction, Lorentz-Fitzgerald, Fitzgerald-Lorentz, physical contraction, contraction factor, gamma factor, aether, luminiferous aether, absolute motion, Larmor.

* DSSU is the acronym for the Dynamic Steady State Universe - the cosmology theory that holds that aether-space is dynamic and that aether-space expands and contracts regionally and equally resulting in a cosmic-scale cellularly-structured universe. It is a model based on the premise that all things are processes.

A rod moving with the velocity of light would lose its third dimension. It would become a cross-section of itself. ... an electron actually disappears when moving with the velocity of light. -Russian philosopher (Ouspensky, 1931, p360).

\section{Introduction}

"Length contraction" refers to two phenomena. One is the apparent change of length as a consequence of relative motion as formulated by Einstein's special relativity theory. The other is the very real change of length as a consequence of absolute motion through aether-space as formulated by DSSU* theory. The present article explores the latter.

But let us first be clear about the differences between the two types of length contraction.

In Einstein's special relativity theory (ESR), the "length" that is subjected to length contraction is that of an object or to any measurable distance such as the empty space between two particles, objects, or astronomical bodies. The "length" refers to any dimension which is axial, or parallel, to the direction of motion. In the DSSU interpretation, the length refers exclusively to the dimension of physical particles, objects, or astronomical bodies. Empty spaces are contracted only to the extent to which they are intrinsically part of the affected object. And, again, it is only those dimensions which are axial to the direction of motion that are affected.

The degree of length contraction, under ESR theory, depends entirely on the relative motion of the observer (which is equivalent to saying "the relative motion of the object"). Thus, the same object will appear to have different lengths for different viewers with different motion. In contrast, in the physical theory, the length is uniquely determined by the speed through aether-space.

For ESR theory, length contraction is considered real only in the sense of being observable. But because it is observer dependent it cannot be considered a physical contraction (unless the observer happens to be at rest with respect to the aether medium). For DSSU theory, length contraction, associated with intrinsic motion, is fundamentally physical, although extremely difficult to observe. 
Einstein's phenomenon requires relative motion. The physical phenomenon, on the other hand, requires NO relative motion. It requires absolute motion through the aether medium.

Why has it taken so long to recognize the phenomenon of intrinsic contraction? The problem is that physical length contraction cannot be measured directly. One cannot take a measuring stick (such as a standard meter rod) or a carpenter's flexible tape and lay it along-side, say, a speeding space-capsule to determine how much the capsule has shrunk in length. All measuring devices, physical or optical, undergo the same fractional amount of contraction as the object of interest.

However, the speed of motion through aether-space can be measured (Miller, 1933; Cahill, 2006). Once the speed is known, it can then be used to calculate the contracted length of the speeding capsule.

In the following sections, the length contraction process will be described and the length contraction factor will be derived. We begin with an aquatic-orbit analogy.

\section{Nautical Orbit Analogy}

\subsection{Essential Conditions}

We will use a motor boat circumnavigating a channel marker as a simple analogy for an electron orbiting a nucleus. The boat will be traveling (on a river) at a constant speed with respect to the water.

The essential conditions for a stable and symmetrical orbit are (1) $x$-axis mirror symmetry and $y$-axis mirror symmetry, and (2) the total time-of-travel parallel to the river MUST EQUAL the total time-of-travel perpendicular to the river.

Examples of symmetrical orbits are shown in Figure 1.
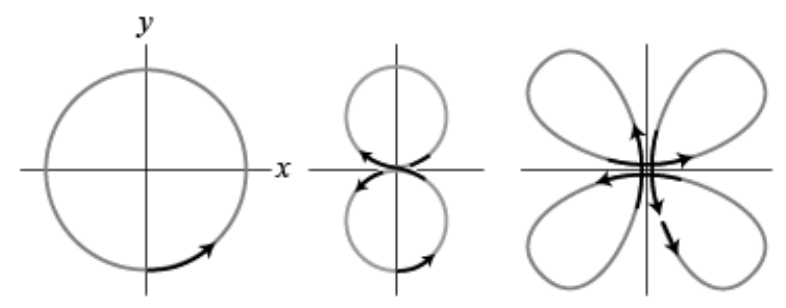

Figure 1. Examples of orbits, in a plane, having both $x$-axis and $y$-axis mirror symmetries

Let us express the travel-time condition in the form of an equation:

$$
(\text { Total time } x \text {-direction })=(\text { Total time } y \text {-direction }) \text {, }
$$

which we abbreviate to, $T_{x}=T_{y}$.

These two conditions (along with the constant speed of the boat) ensure that the circumnavigation will start and end at the very same spot and do so at regular time intervals - regardless of the speed of the river current. It is assumed that the speed of the river flow is constant and less than the speed of the boat. In terms of the electron, the conditions ensure a stable orbit.

Initially, it is assumed that the river is absolutely still—no water flowing in either direction.

Now, the most useful way to express the time condition is in the form of the sum of vanishingly small time increments (see Figure 2):

$$
\Sigma d t_{x}=\Sigma d t_{y} .
$$




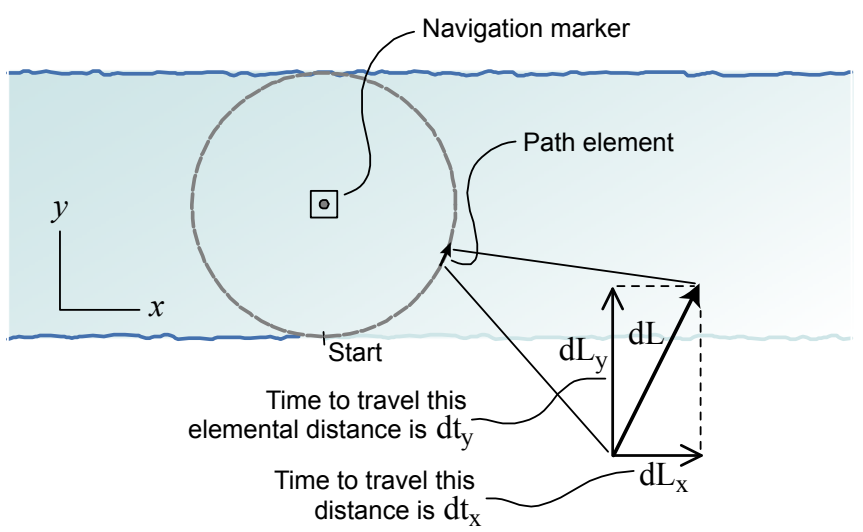

Figure 2. The round-trip path can be divided into elemental distances (each split into its $x$ and $y$ components). "Condition 2" (see text) involves a summation of all the elemental travel times, $d t_{x}$, in the $x$ direction and another summation for those, $d t_{y}$, in the $y$ direction

Let us consider a circular trip around the navigation marker shown in the drawings. From the vectors detailed in Figure 3 (a) we obtain the general expression for the time increment corresponding to the $x$-axis, $d t_{x}=\left(D_{\mathrm{x}} / 2 v\right) d \theta$. By integrating (summing) this expression, going from the starting point (where $\theta$ is $\left.-\pi / 2\right)$ to the finishing point (where $\theta$ is $+3 \pi / 2$ ), the corresponding total time is found:

$$
\begin{gathered}
T_{x}=\sum d t_{x}=\int_{-\pi / 2}^{3 \pi / 2} \frac{D_{x}}{2 v} d \theta, \\
T_{x}=\pi D_{x} / v
\end{gathered}
$$

Similarly, from the vectors detailed in Figure 3 (b) we obtain the general expression for the time increment corresponding to the $y$-axis, $d t_{y}=\left(\mathrm{D}_{\mathrm{y}} / 2 v\right) \mathrm{d} \theta$. And by integrating, from $\theta=-\pi / 2$ to $\theta=(+3 / 2) \pi$, the corresponding total time is found:

$$
\begin{gathered}
T_{y}=\sum d t_{y}=\int_{-\pi / 2}^{3 \pi / 2} \frac{D_{y}}{2 v} d \theta, \\
T_{y}=\pi D_{y} / v .
\end{gathered}
$$

Apply the time equality condition, whereby $T_{x}$ must equal $T_{y}$, and we find, as expected,

$$
\begin{aligned}
\pi D_{x} / v & =\pi D_{y} / v, \\
D_{x} & =D_{y} .
\end{aligned}
$$

Of course, this simply means that no length contraction has occurred.

A simple check on the analysis: Total trip time is $T_{x}+T_{y}=\pi D_{x} / v+\pi D_{y} / v=2 \pi D / v$ (because $D_{x}=D_{y}=D$ ). And agrees with the conventional expression, (trip time) $=$ (circumference) $/$ (speed). So, even if the reader is not familiar with calculus, the procedure obviously gives the correct travel-time formula. 

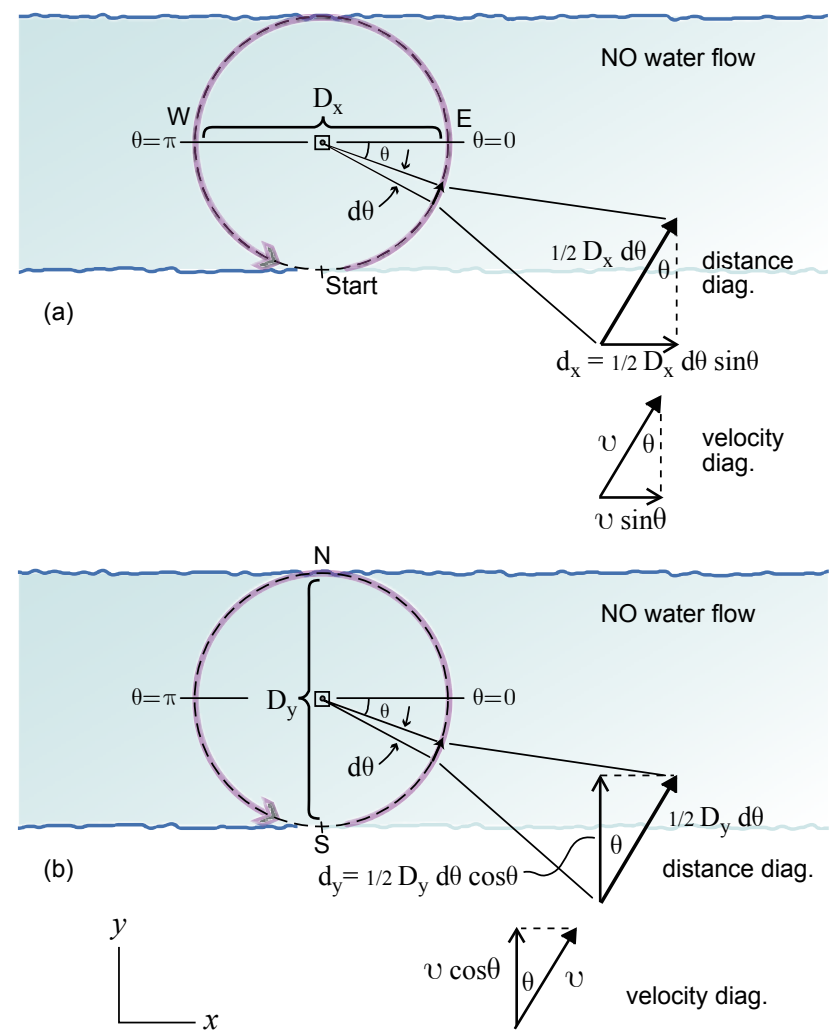

Figure 3. Distance and velocity components for the boat trip are shown. The corresponding travel time, of course, is distance divided by speed. Thus, in part (a), $d t_{x}=d x / v_{x}=\left(1 / 2 \mathrm{D}_{\mathrm{x}} \sin \theta \mathrm{d} \theta\right) /(v \sin \theta)$, which simplifies to $d t_{x}=\left(\mathrm{D}_{\mathrm{x}} / 2 v\right) \mathrm{d} \theta$. Similarly, in part (b), $d t_{y}=d y / v_{y}=\left(1 / 2 \mathrm{D}_{\mathrm{y}} \cos \theta \mathrm{d} \theta\right) /(v \cos \theta)$, which simplifies to

$$
d t_{y}=\left(\mathrm{D}_{\mathrm{y}} / 2 \mathrm{v}\right) \mathrm{d} \theta
$$

\subsection{Adding Flow to the Medium of Our Analogy}

Next, we apply the two conditions to an "orbit" on a flowing river. The speed of the current is $v_{\mathrm{r}}$.

From the perspective of an observer standing on the shore of the river the apparent speed of the boat is $\left(v+v_{r}\right)$ when it is travelling downstream and $\left(v-v_{\mathrm{r}}\right)$ when it is travelling upstream. As before, $v$ is the constant speed of the boat (with respect to the water). And be reminded of the restriction mentioned above, the speed of the river is less than the speed of the boat.

Let us sum the time increments for the $x$-directions. Since the velocity in the positive- $x$ direction is different from the one in the negative- $x$ direction, it is best to do the sum in two parts.

For the semicircular trip from East to West we determine, as shown in Figure $4(\mathrm{a})$, that $d t_{x^{-}}=\left(1 / 2 \mathrm{D}_{\mathrm{x}} \mathrm{d} \theta\right) /\left(v+v_{\mathrm{r}}\right)$. This expression will be integrated from the East point (where $\theta$ is zero) to the West point (where $\theta$ is $\pi$ ) to obtain the travel time for the negative- $x$ direction.

And for the semicircular trip from West to East, for which the applicable speed component is $\left(v-v_{\mathrm{r}}\right)$, we determine, as shown in Figure $4(\mathrm{~b})$, that $d t_{x^{+}}=\left(1 / 2 \mathrm{D}_{\mathrm{x}} \mathrm{d} \theta\right) /\left(v-v_{\mathrm{r}}\right)$. Then by integrating from $\mathrm{W}$ (where $\theta$ is $\pi$ ) back to $\mathrm{E}$ (where $\theta$ is $2 \pi$ ), we find the travel time for the positive- $x$ direction. 


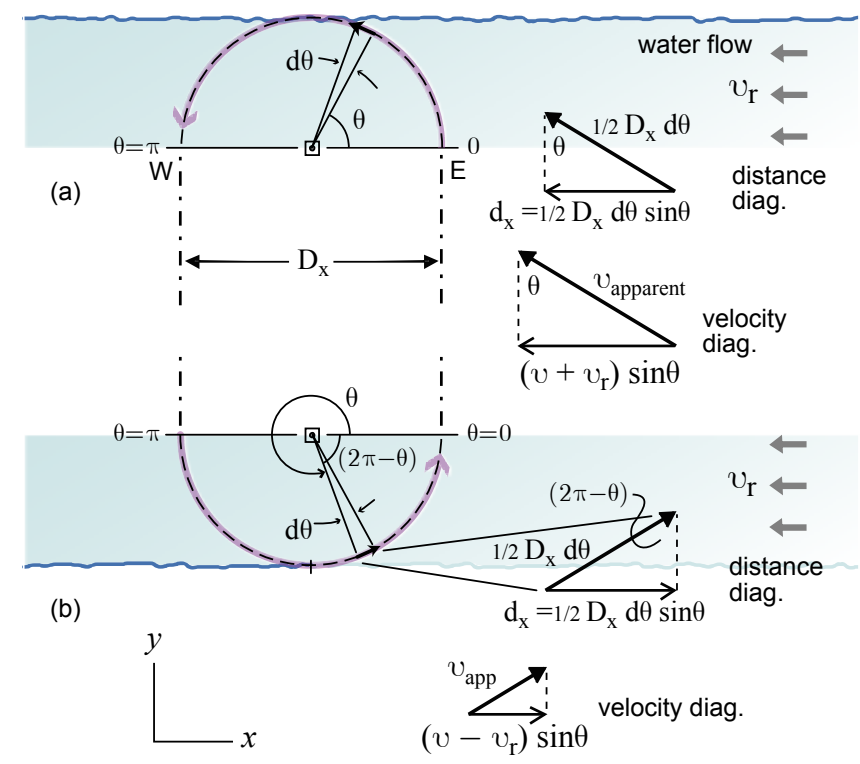

Figure 4. Part (a) gives the motion components for the upper portion of the trip, from $\mathrm{E}$ to $\mathrm{W}$. These components give us the $\Delta$ time expression for the negative- $x$ direction: $d t_{x^{-}}=\left(1 / 2 \mathrm{D}_{\mathrm{x}} \sin \theta \mathrm{d} \theta\right) /\left(v+v_{\mathrm{r}}\right) \sin \theta$, which simplifies to $d t_{x^{-}}=\left(1 / 2 \mathrm{D}_{\mathrm{x}} \mathrm{d} \theta\right) /\left(\mathrm{v}+v_{\mathrm{r}}\right)$; Part (b) gives the motion components for the lower portion of the trip, from $\mathrm{W}$ to $\mathrm{E}$.

These components give the $\Delta$ time expression for the positive- $x$ direction: $d t_{x+}=\left(1 / 2 \mathrm{D}_{\mathrm{x}} \sin \theta \mathrm{d} \theta\right) /\left(v-v_{\mathrm{r}}\right) \sin \theta$, which simplifies to $d t_{x^{+}}=\left(1 / 2 \mathrm{D}_{\mathrm{x}} \mathrm{d} \theta\right) /\left(v-v_{\mathrm{r}}\right)$

The total $T_{x}$ time is calculated as follows,

$$
\begin{gathered}
T_{x}=\sum d t_{x-}+\sum d t_{x+} \\
T_{x}=\int_{0}^{\pi} \frac{D_{x}}{2\left(v+v_{r}\right)} d \theta+\int_{\pi}^{2 \pi} \frac{D_{x}}{2\left(v-v_{r}\right)} d \theta \\
T_{x}=\frac{\pi D_{x}}{2\left(v+v_{r}\right)}+\frac{\pi D_{x}}{2\left(v-v_{r}\right)} \\
=\frac{\pi D_{x} v}{\left(v^{2}-v_{r}^{2}\right)}
\end{gathered}
$$

We must now find the y-direction travel time, $T_{\mathrm{y}}$.

But what is the speed component for the $y$-direction? From the perspective of an observer standing on the shore of the river (or even better, looking down from a bridge) the apparent speed of the boat when the boat is travelling perpendicular to the shoreline is $\sqrt{ }\left(v^{2}-v_{\mathrm{r}}^{2}\right)$. See Figure 5. And, in connection with the boat's "orbit", this speed occurs ONLY at points E and W, for only at these two points is the path directed perpendicular to the shoreline. At all other points, the magnitude is less. At all other points, the magnitude of this component is diminished by the factor $\cos \theta$. 


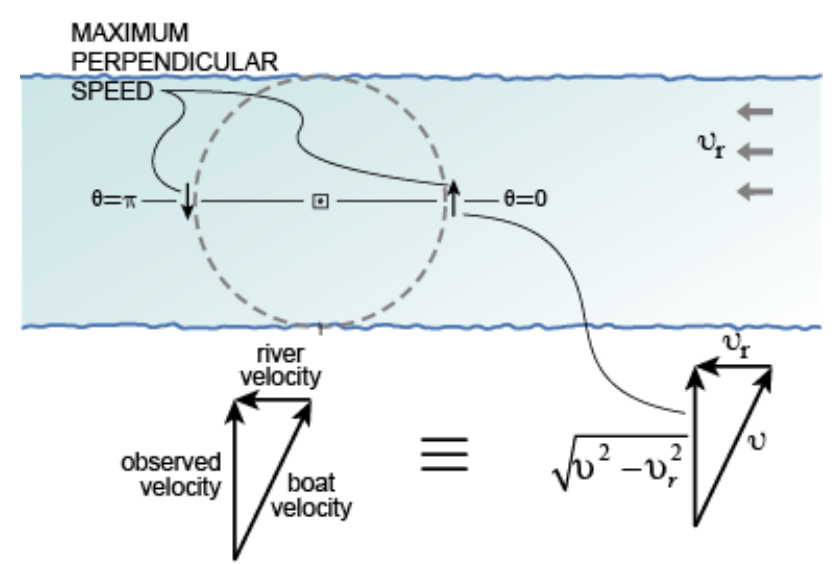

Figure 5. Maximum $y$-component speed occurs at the instant the boat coincides with the east or west points. It is given by the Pythagorean Theorem applied to the right-angled triangle formed by the boat-, river-, and observedvelocities. At all other points on the orbital path, the $y$-component speed is given by, $\sqrt{(}\left(v^{2}-v_{\mathrm{r}}^{2}\right) \times \cos \theta$

The elemental time-increment for the $y$-direction (see Figure 6) is,

$$
d t_{y}=\frac{1 / 2 D_{y} \cos \theta}{\sqrt{v^{2}-v_{r}^{2}} \cos \theta} d \theta ;
$$

which simplifies to,

$$
d t_{y}=\frac{D_{y}}{2 \sqrt{v^{2}-v_{r}^{2}}} d \theta
$$

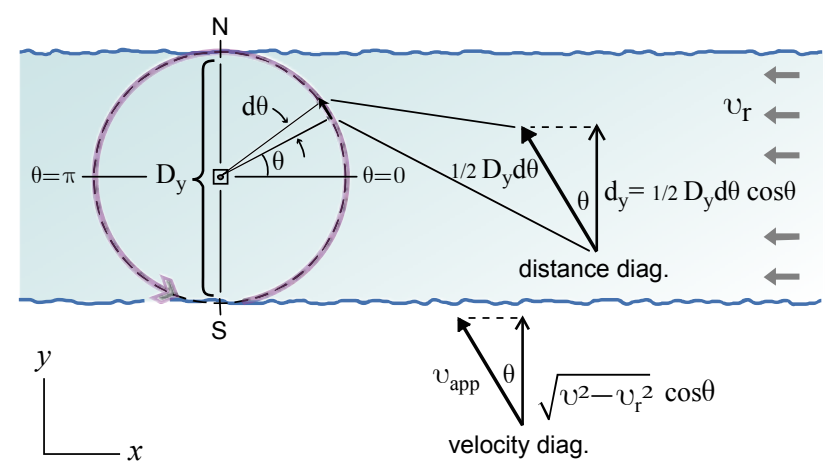

Figure 6. Maximum cross-stream speed is $\sqrt{v^{2}-v_{r}^{2}}$; but varies according to one's position on the orbit. Thus, $d t_{y}$ is given by the distance $\left(1 / 2 \mathrm{D}_{\mathrm{y}} \mathrm{d} \theta \cos \theta\right)$ divided by the speed $\left(\sqrt{v^{2}-v_{r}^{2}} \cos \theta\right)$

The total $T_{y}$ time, for the full round trip, is then found by integrating from $\theta=-\pi / 2$ to $\theta=(+3 / 2) \pi$, as follows,

$$
\begin{gathered}
T_{y}=\sum d t_{y}=\int_{-\pi / 2}^{3 \pi / 2} \frac{D_{y}}{2 \sqrt{v^{2}-v_{r}^{2}}} d \theta \\
=\frac{\pi D_{y}}{\sqrt{v^{2}-v_{r}^{2}}} .
\end{gathered}
$$

Finally, we apply the time equality condition, whereby $T_{x}$ must equal $T_{y}$, that is, 


$$
\frac{\pi D_{x} v}{\left(v^{2}-v_{r}^{2}\right)} \text { must equal } \frac{\pi D_{y}}{\sqrt{v^{2}-v_{r}^{2}}}
$$

Solving for $D_{x}$ we find,

$$
D_{x}=\sqrt{1-v_{r}^{2} / v^{2}} \times D_{y} \cdot \quad v_{\mathrm{r}}<v
$$

What this means, unequivocally, is that to comply with the condition $T_{x}=T_{y}$, and have the boat complete its constant speed orbit, the $x$-direction "diameter" MUST SHRINK. And that shrink factor is $\sqrt{1-v_{r}^{2} / v^{2}}$. If, $v$, the speed of the orbiting object, is held fixed, then the magnitude of the factor depends entirely on $v_{\mathrm{r}}$ the speed of the medium.

The faster the river flows, the more pronounced is the diameter contraction. The orbit becomes oblate. See Figure 7.

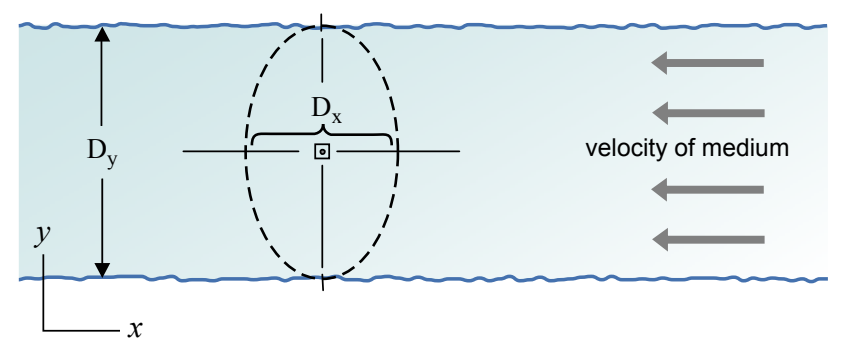

Figure 7. The "orbit", when constrained by the condition described in the text, will be deformed to an elliptical shape by the flow of the medium. Those dimensions that are parallel to such flow will undergo length contraction

Turning now to atomic-scale length contraction.

\section{Electron, Electron Orbit, and Orbit Contraction}

\subsection{Nature of the Electron}

There are three things, regarding the electron and its orbit that are of particular interest to the discussion and should be clarified.

(1) The electron is not a point particle.

(2) The speed of the electron is always less than the speed of light; however, its sole sub-component IS moving at the full speed of light.

(3) The stability of the electron's orbit about the atomic nucleus, regardless of actual trajectory, is still definable by the conditions set out in Section 2 above.

Although the electron is classified as a fundamental particle, it is actually composed of a single sub-unit. The electron is simply a confined photon. That is to say, the electron is a photon travelling in a tightly bound pattern. A simple analogy might that of a dog chasing its tail; but instead of a circular path, there is an additional loop. The electron is a photon whose pattern of propagation is along a twisted helical loop of sub-atomic scale (Hu, 2004). It turns out that the electron is a photon with toroidal topology (Williamson \& van der Mark, 1997).

In fact, an important premise of DSSU theory is that ALL particles are the manifestations of one or another configuration (and there are many possible patterns) of photon confinement. (Ranzan, 2012; Williamson \& van der Mark, 1997)

The electron subunit is conducted by the aether medium. (We conceptually jump from a boat being 'conducted' over a watery medium, to a photon being conducted by an aether medium.) And the fact that the photon is a confined photon does not in any way change its speed with respect to the aether. It is always $c$.

While the electron is a confined photon—which always travels at light-speed-the electron itself has a wide 
range of travel speeds. For instance, the electron can be accelerated very close to the speed of light, but it can also be held stationary within a so-called magnetic bottle. That is, an electron may be stationary while its internal photon is whizzing around at the astounding speed of light!

An electron traveling through a conductor as part of an electric current has a significant and highly erratic instantaneous velocity and instantaneous kinetic energy. But it is only the very small net motion that is important. This net motion is known as the "drift velocity" (amazingly it's magnitude is only a fraction of a millimeter per second!) and account's for the electron's correspondingly small net kinetic energy (Benson, 1991).

Essentially the actual path of the electron in its chaotic dance matters not in the least to ordinary electric phenomena. It is the net path that conveys the electric current along a conductor.

Similarly, the actual paths of the confined photons that constitute all matter is not important. What is important is the net motion in 3-space. For instance, consider an atom at rest in aether-space. The sum of the algebraic distances that the electron (or its confined photon) travels - no matter how vigorously - will be a zero sum, on average. Geometrically this means that the algebraic sum of the motions in the $x$-direction is zero; as is the sum in the $y$-direction and the $z$-direction.

The average distance that the electron's actual path deviates from such zero point provides a crude representation of the average radius of the electron's orbit.

Incidentally, the reader may be wondering where is the electron's confined photon going at the enormous speed of light? - after all, the electron may simply be sitting there in an arrested state in a vacuum? The answer is that the electron has a property known as spin. All electrons are classed as "spin 1/2" particles. Most of the motion of confined photon is manifest in the electron spin. The photon is involved in its own helical, self-orbiting, motion - 'tail chasing' with a toroidal twist. (What about the zero-spin particles? The pion, for instance, is said to have zero spin. It is the same story; there is some form of self-orbital motion. Realize "zero" spin does not mean that the particle is not spinning; it simply means the NET spin is zero.)

\subsection{Electron Orbit}

The point I want to stress is that the orbital path of an electron (about the atomic nucleus), although it might be quite elaborate, contains the even more detailed path (but hidden) of its confined photon sub-unit.

Consider a highly simplified electron orbit (Figure 8 (a)). Imagine the tangled path of the electron's sub-unit. The mind's picture should include spirals superimposed on a wave pattern and look something like the complex spiral patterns shown in Figure 8 (b).

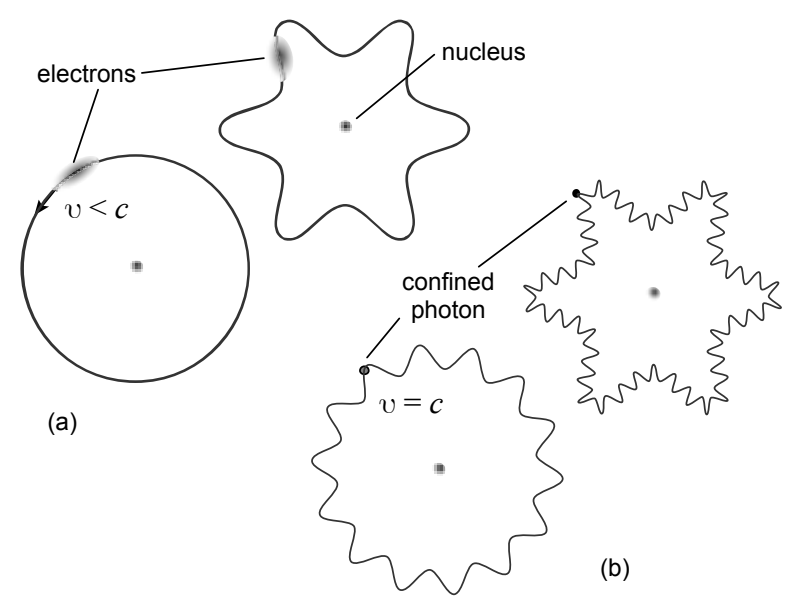

Figure 8. Part (a) shows two examples of a simplified orbital path of the electron as it orbits the nucleus. Part (b) shows the far more complex paths of the sub unit - the confined photon - that constitutes the electron. Note that the electron's speed varies with the electromagnetic force; but the confined photon's speed is always constant

Now the problem is that we do not know the precise speed of the electron as it orbits the nucleus. Like a comet near the Sun, the electron quickens as it nears the proton(s) but moves comparatively slowly at the extremities of its orbit. However, we do know the speed of the confined photon. We know it exactly. (We also know that electron orbits have symmetry.) 
So here is the problem in a nutshell: We know (at least nominally) the path of the electron, but not its speed. We know the speed of the electron's sub-unit but not the path.

The solution is to simply unwind the waving-and-spiraling trajectory of the confined photon and to form a stretched-out smooth circle - forming a new and larger circular orbit. See Figure 9. The 'confinement' photon, and this is the key point, follows this thought-experiment orbit with a constant speed of $c=300,000 \mathrm{~km} / \mathrm{s}$ (with respect to aether).

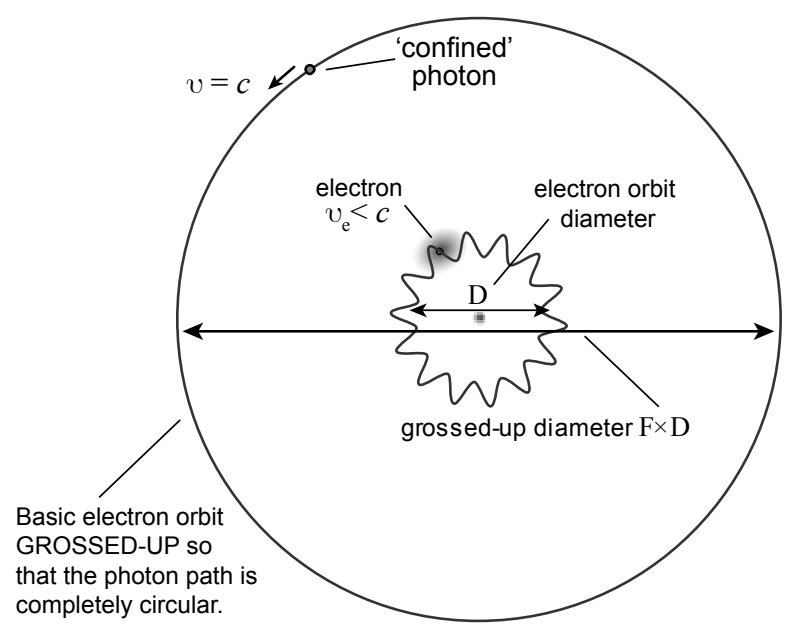

Figure 9. The electron's orbit, regardless of complexity of shape, is here schematically stretched so that the electron's 'confined' photon is orbiting the atomic nucleus along a perfectly smooth circular path -a path that the photon necessarily travels at the speed of light. "F" stands for the factor required to conceptually gross-up the diameter $D$ to achieve the stretched-out path

What about the diameter of this grossed-up orbit? From the nominal symmetry of the electron orbit we obtain a nominal diameter $D$; we then apply a gross-up factor $F$; the result is the grossed-up diameter $F \times D$. (The value of the factor is not important for determining length contraction. It conveniently cancels out of the equation.)

\subsection{Contraction of the Electron Orbit}

A quick rundown of the assumptions: The aether flow is collinear with the $x$-axis. The subunit of the electron is conducted by the aether medium with a constant speed $c$.

A quick review of the essential conditions for a stable orbit: The electron orbit has mirror symmetry on both axes. During each orbit the total time-of-travel parallel to the aether flow MUST EQUAL the total time-of-travel perpendicular to the flow.

If an electron does not return to its starting point (contrary to our assumption) it can only mean that the symmetry has been lost and the energy level has changed - reflecting a momentary instability in the atom. But since we are assuming a stable atom with a stable electron orbit, the electron does return to an idealized initial point.

The analysis leading to the length contraction equation is identical to the one for the 'orbit' on a flowing river described earlier except for the identity of the variables and the addition of the gross-up factor. Note, in particular, that in the present situation the speed of the entity doing the orbiting is $c$ instead of $v$; while the speed of the medium is now $v_{\text {aether. }}$ See Figure 10. 


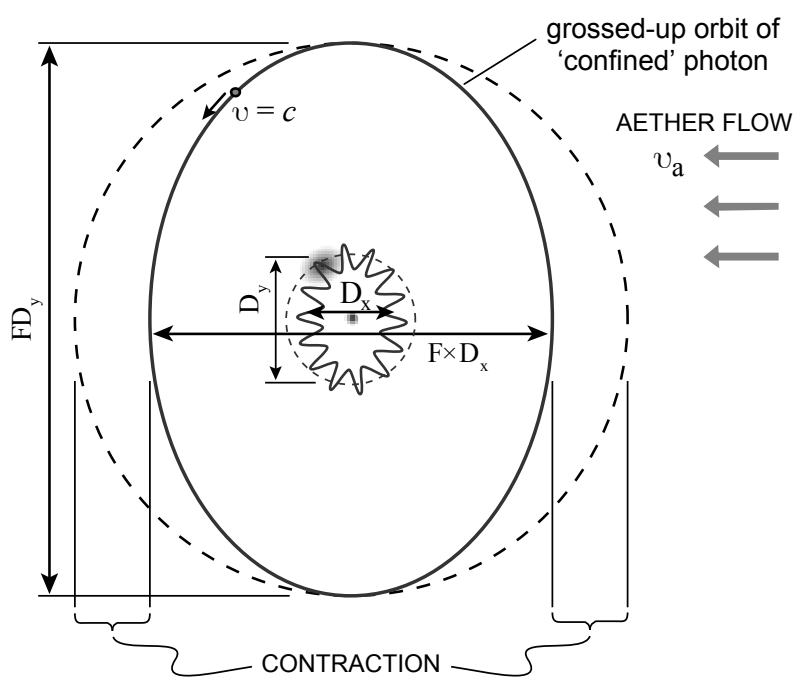

Figure 10. Aether flow causes a circular electron orbit to become elliptical; and (in three dimensions) causes a spherical electron cloud to become oblate

For each orbit, the total travel time parallel to the aether flow $v_{\mathrm{a}}$ is,

$$
T_{\|}=\sum d t_{x}=\frac{\pi F D_{x} c}{\left(c^{2}-v_{a}^{2}\right)} .
$$

And the total travel time perpendicular to the aether flow $v_{a}$ is,

$$
T_{\perp}=\sum d t_{y}=\frac{\pi F D_{y}}{\sqrt{c^{2}-v_{a}^{2}}} .
$$

Apply the key condition. The subunit (and the electron) spends the same amount of time travelling parallel to the $x$-axis as it does parallel to the $y$-axis. (The actual direction, of course, is continually changing).

$$
\begin{aligned}
T_{\|} & =T_{\perp}, \\
\frac{\pi F D_{x} c}{\left(c^{2}-v_{a}^{2}\right)} & =\frac{\pi F D_{y}}{\sqrt{c^{2}-v_{a}^{2}}} .
\end{aligned}
$$

Solving for $D_{x}$ we find,

$$
D_{x}=\sqrt{1-v_{a}^{2} / c^{2}} \times D_{y} \cdot \quad v_{\mathrm{a}}<v
$$

The radical $\sqrt{1-v_{a}^{2} / c^{2}}$ is the contraction factor. And since whenever there is aether flow, its value is always less than unity, $D_{\mathrm{x}}$ must be less than $D_{\mathrm{y}}$. Clearly the $x$-direction diameter MUST SHRINK - the unavoidable result of Nature's imposed conditions.

The faster the aether flows the more pronounced is the diameter contraction. An otherwise circular orbit becomes flattened; an otherwise spherical electron cloud becomes oblate. See Figure 10.

If an object could travel at the speed of light, then the extreme situation would arise. The object would then, as described by the Russian philosopher P. D. Ouspensky (in the opening quote), lose its third dimension and become a cross-section of itself. Its electrons would actually disappear. And the confined photons would cease their self-orbiting. 


\section{Macro-Structure Contraction}

\subsection{Atomic and Molecular Realm}

In the realm of the atom, because of the uncertainty principle, the orbiting electrons are viewed less as point particles than as electron clouds - regions centered on the atom's nucleus. These clouds take on a variety of shapes, all of which are subject to the same shrinkage just described. All become flattened in the direction of motion, in proportion to the absolute speed, according to the contraction factor.

Whatever forces bind atoms together, to form molecules, crystals, and ordinary solids, will continue to do so as the aether flow increases. Normally spherical atoms become oblate. Molecules become flattened. Objects, including the standard rulers we use to measure lengths, embody their micro-structure and undergo what we call physical contraction (Figure 11). The degree of contraction depends entirely on the object's motion through aether - or simply the speed of the aether wind.

For dimensions collinear with the aether flow the contraction factor is $1 / \gamma$.

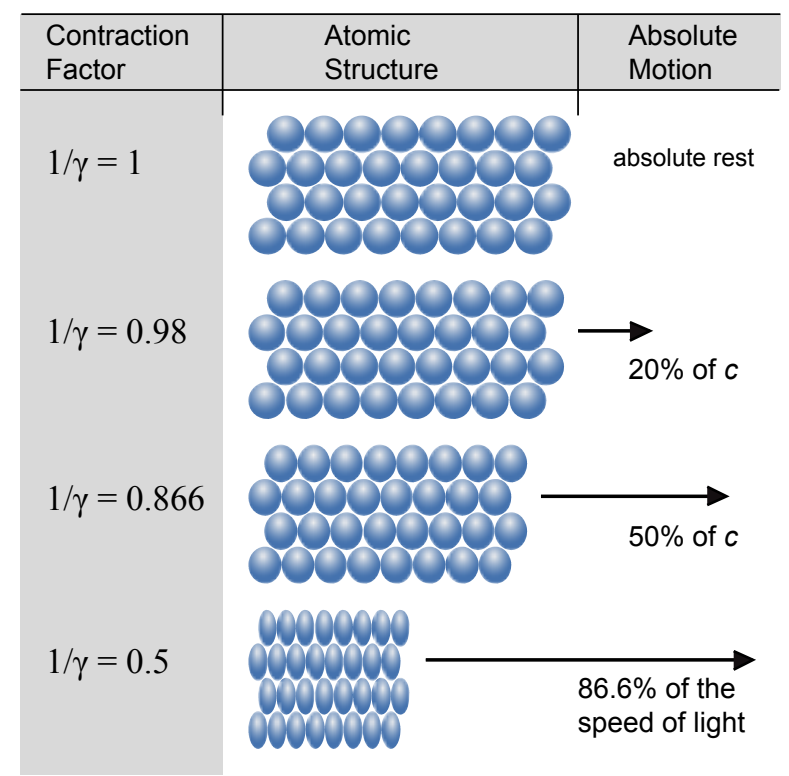

Figure 11. Idealized atomic structure is contracted by varying amounts - but only in the direction of absolute motion

\subsection{The Gamma Expression}

For convenience the physical contraction factor $\sqrt{1-v_{a}^{2} / c^{2}}$ may be expressed as $1 / \gamma$. We may thus refer to the gamma term as,

$$
\gamma=\frac{1}{\sqrt{1-v_{a}^{2} / c^{2}}} .
$$

This expression should be familiar to students of Einstein's relativity theory (except that in his theory $v_{a}$ would refer to the relative motion, and not the absolute motion).

\subsection{Not Directly Observable}

In a hypothetical situation of physical length contraction, no matter how extreme, the contraction effect would not be directly observable. There is a simple and reasonable argument that explains why.

Consider a square object and a round object resting on a table. Initially there is no aether flow and no contraction. If one looks down on each object, in "plan" view, a corresponding image will be produced on the retina of the eye (Figure 12 (a)). Now, using thought-experiment empowerment, we turn "on" the aether flow and induce extreme lateral contraction as shown in Figure 12 (b). The square object becomes rectangular, the circular object 
becomes oval, and the eyeball becomes oblate.

The eye's ciliary muscles will, of course, continue to automatically adjust the curvature of the eye's lens to bring the image into focus on the retina. The ciliary muscles will focus a contracted image onto a contracted retina, activating the same light receptors that had been activated prior to the introduction of a distorting aether-flow. Since the same light receptors would be activated in the retina, the brain would continue to interpret each object shape as a perfect undistorted square and a normal circular disk (Figure 12 (b)).

Although not directly observable, physical contraction is indirectly detectable as discussed briefly in Section 6 .
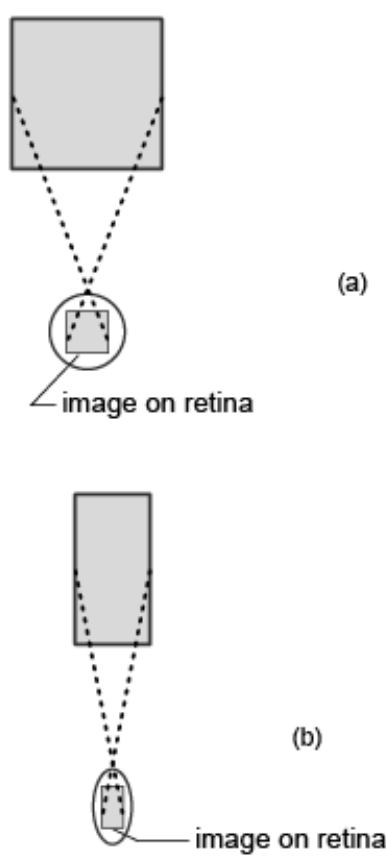

(a)
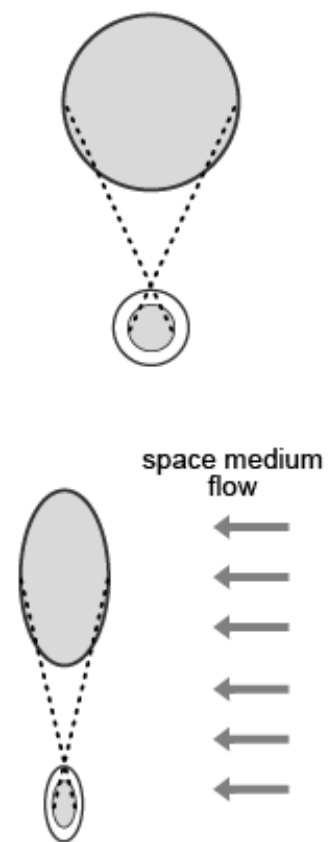

Figure 12. Contraction thought experiment. (a) Objects observed in "plan" view in the absence of length contraction. (b) The same objects with extreme lateral contraction produce a contracted image within a contracted eyeball. The expectation is that the same light receptors would be activated in the retina, for both upper and lower situations, so that the brain would not recognize any significant difference

\subsection{Non-Physical Length Contraction}

In the physics of relative motion there is another type of contraction-length contraction that is observer dependent and requires relative motion between object and observer. Interestingly, the contraction expression is almost identical (yet the meaning is fundamentally different). An observed length of a moving object is equal to the contraction factor times the "rest length" of that object (that is, the length of the object measured within the objects own frame of reference).

$$
D=(1 / \gamma) \times D_{o} .
$$

Variance in apparent length, under conditions of relative motion, is a consequence of the need to measure simultaneously the two extremities of an object. The two acts of measuring are the two events that enter into the Lorentz transformation equation. The problem is that two events, simultaneous for one observer, need not be simultaneous for another! It all depends on the relative motion of the observer. And so, different observers (with different relative motion) will "measure" different lengths for the very same object.

The ESR formalism ignores the aether and deals only with the apparent length contraction, which contraction is not necessarily real since it varies for different inertial observers.

When comparing Einstein's version with the author's thesis, the essential point is this: ESR's length contraction depends only on relative motion; while intrinsic contraction depends only on motion with respect to the aether space-medium. 


\section{Orbit-Length versus Orbit-Period}

What if physical contraction were a false concept? What if it were the orbit length that remains constant instead of the orbit period? Wouldn't the contraction idea then be unnecessary?

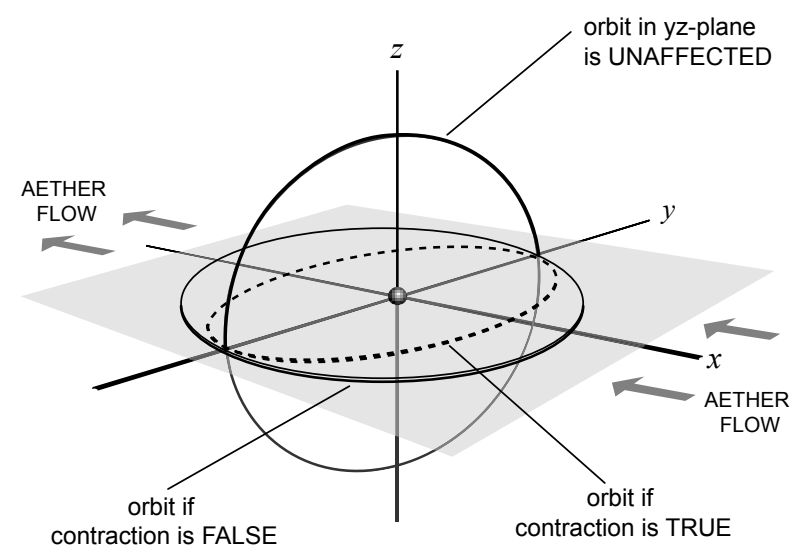

Figure 13. The reason behind length contraction is to make the period of the elliptical orbit equal to the period of the circular ( $y z$-plane) orbit. However, if one denies the validity of 'contraction', then it becomes impossible to have the period of the uncontracted ( $x y$-plane) orbit equal to the period of the unaffected ( $y z$-plane) orbit! Such atom would self-destruct. The choice is between orbit-time equality or orbit-length equality

First, realize this important feature of the contraction effect: The electron orbit in the plain of motion becomes elliptical while the perpendicular orbit remains unaffected (remains circular). In other words, if the elliptical orbit is rotated on the $y$-axis, it becomes circular (and is no longer subject to the contraction effect). See Figure 13. Recall, that it was the time-equality condition that gave us the elliptical orbit in the first place; when the orbit is rotated, that time-equality does not change. Furthermore, it can easily be shown that the period of the elliptical orbit is equal to the period of the rotated orbit (now circular in the $y z$-plane).

Now assume, for the sake of argument, the concept of physical contraction is false. Say, one rejects that the plane-of-motion orbit becomes elliptical, and instead, claims that it remains circular. Then one must alternatively, if objective reality has any meaning, accept that the plane-of-motion orbit (since it now remains circular and therefore has a longer path, longer than the elliptical path) takes longer in time. It must have a longer period than the corresponding elliptical orbit; and also a longer period than a perpendicular orbit (in the $y z$ plane, Figure 13), which was unaffected by the contraction effect. But such period-variation would lead to serious consequences. The various orbits within an atom would not be in a synchronized state. Orbital periods would change according to orientation with respect to the axis of the atom's motion (or direction of aether flow). Such atom would be in an unstable chaotic state. The electron(s) would either collapse into the nucleus or fly off.

Electrons have a wave-particle duality and exhibit both wave and particle phenomena. Electrons travel as waves. A quantum rule in physics requires that the electron orbit must consist of an integer number of wavelengths (Figure 14a). If the period of a conforming orbit is altered, then the same number of wavelengths will no longer fit that same orbit—-jeopardizing compliance to the quantum rule. See Figure 14b.

Thus, the denial of length contraction leads to variation in orbit time which in turn leads to a violation of the quantum rule. 


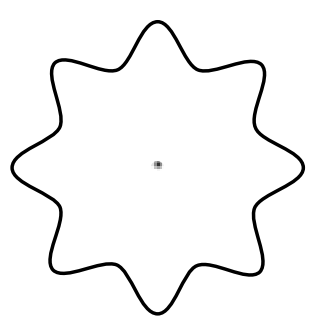

(a)

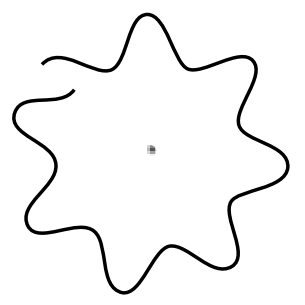

(b)

Figure 14. (a) Quantum physics demands that the electron orbit must accommodate an integer number of wavelengths. (b) If the orbit period were not constrained, then a totally improbable orbit would be predicted

By allowing orbit length to vary (in accordance with the discussed length-contraction effect) and the orbit time to remain fixed (regardless of orbit orientation), electron orbits are able to maintain their quantum stability.

The main point is that a change in the orbit orientation does not change the orbit time - courtesy of the length contraction effect.

However, a change in the aether speed DOES change the orbit time - the greater the aether speed the longer it takes to complete an orbit. This is so for all orientations of the orbit. And therein lies the deep connection between real length-contraction and real clock-slowing; the two phenomena go hand in hand; both are induced by motion with respect to the space medium.

\section{Historical Notes, Evidence, and Reflections}

\subsection{Historical Note: Larmor}

Early prediction of length contraction. In 1900, Larmor considered a system "composed of two electrons of opposite charge" (one would say today: composed of an electron-positron pair). He neglected irradiation, and assumed circular orbits about the common center-of-mass of the two particles. Assuming also that the whole system was in motion through aether, he proved that the velocity dependent deformation of the electric fields predicted by classical physics generated, in the bound system, exactly the contraction postulated by Fitzgerald and Lorentz. (Selleri, 2004)

What this means is that ANY bound system of particles is predicted to contract. All subsystems are predicted to contract, including protons, neutrons, mesons, and other subatomic particles.

\subsection{Historical Note: Sir Arthur Eddington}

Eddington, the foremost promoter of Einstein's relativity theory, said that the radius of the electron orbit must adjust itself at each point of space so that measuring rods, which are composed of electrons, always bear a constant ratio to the radius of curvature of the space at that point. (Hesse, 1961)

In other words, his version of length contraction is related to space curvature. But since no one, as far as I am aware, has ever found a comprehensible physical meaning for "the radius of curvature of space", his version is not a physical concept. It remains solely a mathematical concept (the property of a coordinate system) until someone comes up with a physical connection. Space, empty space, curved or not curved, is not a physical system. Eddington's length contraction (actually his interpretation of Einstein's relativistic effect) is therefore not a physical effect. I'll come back to this in a moment.

\subsection{On the Existence of Aether}

The "aether" that I refer to is the non-material, auto-dynamic, medium that pervades the universe and all interstitial space. More specifically, I refer to the aether of DSSU theory.

Although the existence of aether cannot be denied, it can be ignored, and has been ignored with splendid carefree contempt. Einstein did so. Physics Textbooks continue to do so. The contempt is contagious among science historians as they perpetuate the myth of the null result of the Michelson and Morley experiments, and ignore Dayton Miller's definitive measurements.

The implications of ignoring aether are serious. Ignore aether and you miss an understanding of intrinsic length contraction. Ignore aether and you miss the cause of proven clock retardation. Ignore aether and you have no explanation for why the speed of light is $300,000 \mathrm{~km} / \mathrm{s}$ and not $3,000 \mathrm{~km} / \mathrm{s}$. Ignore aether and you forego the Holy Grail of astrophysics - the causal mechanism of gravitation. Ignore the aether and the cosmic structure of the Universe will not be understood. But most important of all, if you ignore aether, you miss the Primary Cause 
- the physically and philosophically essential cause of all causes.

\subsection{Evidence of Physical Contraction}

Physical length contraction has been historically called Fitzgerald-Lorentz contraction in honor of G. F. FitzGerald and Hendrix A. Lorentz the early developers of the underlying theory.

Their hypothesized contraction is distinctly unlike Einstein's. While Einstein's contraction is NOT real, in that it is entirely dependent on the relative motion of the observer, the Fitzgerald-Lorentz contraction is real.

“... the Fitzgerald-Lorentz contraction is a real dynamical effect of absolute motion, unlike the spacetime view that is merely a spacetime perspective artifact, and whose magnitude depends on the choice of observer."(Cahill, 2005)

Einstein clearly rejected the Lorentzian hypothesis of contraction of the electron when he wrote, "This hypothesis [electron cloud contraction in the direction of motion], which is not justified by any electrodynamic facts, ..." (Einstein, 1952, p57)

Thus is underscored the perils of ignoring aether.

Reginald T. Cahill, in several of his research papers, details how real length contraction affects the Michelson-Morley interferometer. Of particular importance is the M-M interferometer experiment conducted within a vacuum chamber (Cahill, 2004, 2005). The vacuum-mode experiment serves as the definitive proof of physical length contraction (Müller, 2003).

There is a most remarkable insight to be gained from the manner by which matter propagates through space - through aether permeated space. Given that all matter is composed of confined photons (or similar energy particle), which by definition are conducted by the ubiquitous aether, then it follows that a constant aether flow cannot, in any way, resist the constant-speed motion of matter regardless of the magnitude of the aether wind. (It also follows that aether itself cannot be a form of matter - where the meaning of 'matter' encompasses radiation- and mass- particles or entities. Obviously, 'matter' and 'aether' cannot share the same definition! But I'll leave this for a future discussion.)

Just as the aether offers NO resistance to the orbital trajectory of the electron, so too the aether offers NO resistance to the Earth as it orbits the Sun. The Earth and all the other orbiting bodies within our Solar System are quite unaffected by the, more or less, $400-\mathrm{km} / \mathrm{s}$ aether wind ceaselessly streaming through our region of the galaxy.

\section{References}

Benson, H. (1991). Current and Resistance. In University Physics. New York, NY: John Wiley \& Sons, Inc.

Cahill, R. T. (2004). Absolute Motion and Gravitational Effects. Apeiron, 11(1). Retrieved from http://www.orgonelab.org/EtherDrift/CahillApeiron2004.pdf

Cahill, R. T. (2005). The Michelson and Morley 1887 Experiment and the Discovery of Absolute Motion. $\begin{array}{lllll}\text { Progress in } \quad \text { Physics, 25-29. } & \text { Retrieved from }\end{array}$ http://www.mountainman.com.au/process_physics/HPS27.pdf

Cahill, R. T. (2006). The Roland De Witte 1991 Experiment (to the Memory of Roland De Witte). Progress in Physics, 3, 60-65. Retrieved from www.ptep-online.com/index_files/2006/PP-06-11.pdf

Einstein, A. (1952). Relativity The Special and the General Theory (1961 ed.). New York, NY: Wings Books.

Hesse, M. B. (1961). The Theory of Relativity. In Forces and Fields (1965 ed.). New Jersey, Totown: Littlefield, Adams \& Co.

Hu, Q. H. (2004). The Nature of the Electron. Physics Essays, 17, 442-458. http://dx.doi.org/10.4006/1.3025708

Miller, D. C. (1933). The Ether-Drift Experiment and the Determination of the Absolute Motion of the Earth. Reviews of Modern Physics, 5. http://dx.doi.org/10.1103/RevModPhys.5.203

Müller, H. Herrmann, S., Braxmaier, C., Schiller, S., \& Peters, A. (2003). Modern Michelson-Morley experiment using cryogenic optical resonators. Phys. Rev. Lett., 91(2), 020401-1. http://dx.doi.org/10.1103/PhysRevLett.91.020401

Ouspensky, P. D. (1931). A New Model of the Universe (1971 ed.). New York, NY: Vintage Books.

Ranzan, C. (2012). The Fundamental Process of Energy. Publication pending

Selleri, F. (2004). Recovering the Lorentz Ether. Apeiron, 11, 246.

Williamson, J. G., \& van der. Mark, M. B. (1997). Is the electron a photon with toroidal topology? Annales de la Fondation Louis de Broglie, 22(2), 133. Retrieved from www.photontheory.com/ 